\title{
Maternal Mortality Due to an Undiagnosed Large Thymoma in Puerperium: A Case Report
}

\author{
Malihe Fakehi ${ }^{1}$, Sara Saeidi ${ }^{2}$, Maryam Mazloumi ${ }^{1}$, Neda Hashemi ${ }^{3}$, Maryam Rahimi ${ }^{1}$ and Marjan \\ Ghaemi $^{4, *}$ \\ ${ }^{1}$ Shahid Akbarabadi Hospital, Iran University of Medical Sciences, Tehran, Iran \\ ${ }^{2}$ Yas Hospital, Tehran University of Medical Sciences, Tehran, Iran \\ ${ }^{3}$ Rasool Akram Hospital, Iran University of Medical Sciences, Tehran, Iran \\ ${ }^{4}$ Vali-e-Asr Reproductive Health Research Center, Tehran University of Medical Sciences, Tehran, Iran \\ Corresponding author: Vali-e-Asr Reproductive Health Research Center, Tehran University of Medical Sciences, Tehran, Iran. Email: marjan_ghaemi@yahoo.com
}

Received 2021 September 26; Accepted 2021 November 29.

\begin{abstract}
Introduction: Delayed or avoided medical care due to coronavirus disease 2019(COVID-19) related concerns may increase morbidity and mortality associated with both acute and chronic health conditions. Thymoma is uncommon in pregnancy, although it could be accompanied with unfavorable outcomes. We report a puerperal woman presented with dyspnea and cardiac arrest with a recent diagnosis of thymoma that led to maternal mortality.

Case Presentation: A 38-year-old woman with occasional dyspnea during pregnancy without medical referral was admitted to the hospital with severe dyspnea and orthopnea three days after cesarean section. Spiral computed tomography (CT) angiography showed a $64 \times 84$-centimeter mass with soft tissue density in the left perivascular that was originated from the anterior mediastinum; in biopsy, thymoma was suggested. She died shortly after due to severe dyspnea and cardiac arrest.

Conclusions: Prenatal care during COVID-19 pandemic should not be postponed. Indeed, any symptom similar to the physiologic changes in pregnancy needs to be evaluated for optimal clinical management.
\end{abstract}

Keywords: Thymoma, Pregnancy, Puerperium, Maternal Mortality

\section{Introduction}

It is estimated that many people have delayed or avoided seeking for medical care because of concerns about coronavirus disease 2019 (COVID-19) that may lead to higher morbidity and mortality cases associated with both acute and chronic health conditions (1).

Thymoma is the most common primary anterior mediastinum neoplasm accounting for about one percent of all adult malignancies (2). According to the World Health Organization (WHO) criteria, thymoma is classified based on histologic cells (round/epithelioid cells as type A or spindle/oval cells as type $\mathrm{B}$ ) and is divided into A, AB, B1, and B2 subtypes (3).

Patients usually present with mass-associated respiratory symptoms, superior vena cava syndrome, or paraneoplastic syndrome, including myasthenia gravis, pure red cell aplasia, or acquired hypogammaglobulinemia, and connective tissue disorders (4).

Thymoma in pregnancy has unfavorable outcomes with over $90 \%$ mortality during pregnancy or in puer- perium (5). It may not be diagnosed easily due to the overlap with pregnancy-related physiologic changes that may lead to delay or missed diagnosis. There are few published reports assessing thymoma in the postpartum period (6, 7). Herein, we report a case of maternal mortality due to dyspnea and cardiac arrest in puerperium with a recent diagnosis of thymoma.

\section{Case Presentation}

A 38-year-old woman gravida 3 para 2 with two previous cesarean section at 38 weeks of gestational age attended the emergency room of a university hospital affiliated to Iran University of Medical Sciences in February 2020 with labor pain. The vital sign on arrival showed pulse rate (PR) (8) of 90 , respiratory rate (RR) of 22 , blood pressure (BP) equal to $127 / 88 \mathrm{~mm} / \mathrm{Hg}$, and normal temperature. The $\mathrm{O}_{2}$ saturation was $96 \%$ without mask. Evaluation of the upper airway showed no signs suggestive of difficult laryngoscopy, and no hoarseness of voice. Jugular venous pressure was high, and the neck veins were en- 
gorged, that was perused as physiologic volume overload of pregnancy. The results of other physical examinations were unremarkable. She mentioned no previous illness except hypothyroidism in pregnancy that was managed with levothyroxine and denied any history of paraneoplastic diseases. Indeed, she experienced short episodes of dyspnea during pregnancy without any follow up. Her prenatal care was not appropriate due to her fear of being infected with COVID-19.

She was transferred to the operation room, and cesarean section with spinal anesthesia was performed, and a healthy baby girl was born with an Apgar score of 9 and 10 in first and fifth minutes. We recommended her to visit a cardiologist, but she did not accept and was discharged with personal consent. She was readmitted after three days for being tachypneic and dyspneic with RR of 30, orthopnea, and PR of 120 , and decreasing $\mathrm{O}_{2}$ saturation to $82 \%$. Lung auscultation showed sound decreasing in apical region of the left lung. Chest $\mathrm{x}$-ray revealed compression of the heart due to probable cardiac tamponade and obstruction of the superior vena cava and trachea.

She was admitted to the intensive care unit (ICU) with $\mathrm{O}_{2}$ mask, and laboratory test was requested. Her liver function test was abnormal with serum glutamic oxaloacetic transaminase (SGOT) equal to $196 \mathrm{IU} / \mathrm{L}$, serum glutamic pyruvic transaminase (SGPT) equal to $251 \mathrm{IU} / \mathrm{L}$, lactate dehydrogenase (LDH) equal to $1822 \mathrm{U} / \mathrm{L}$, alkaline phosphatase (AlkP) equal to $503 \mathrm{IU} / \mathrm{L}$. The results of the other abnormal laboratory tests were thyroid stimulating hormone (TSH) equal to 4.5, erythrocyte sedimentation rate (ESR) equal to $69 \mathrm{~mm} / \mathrm{hr}$, creatine phosphokinase (CPK) equal to 5484 $\mathrm{IU} / \mathrm{L}$, and creatine kinase-MB (CK-MB) equal to $119 \mathrm{U} / \mathrm{L}$ with abnormal venous blood gases (VBG) suggesting mixed acidosis. However, the d-dimer test was negative. To rule out COVID-19, reverse transcription polymerase chain reaction (RT-PCR) was requested that was negative.

In addition, an immediate consultation with a cardiologist was performed with the suspicion of pulmonary thromboembolism. He requested an electrocardiogram that showed sinus tachycardia. Normal lower limb Doppler ultrasound ruled out deep vein thrombosis (DVT). In echocardiography, a solid nature tumor was detected. Then, a spiral computed tomography (CT) angiography of the thorax was requested, which showed a $64 \times 84$ centimeter soft tissue mass compressing her left lung that was originated from anterior mediastinum (Figure 1).

Therefore, a closed biopsy was suggested, and no evidence of thromboembolism was detected. Ultrasound guided lung mass biopsy revealed bland looking glandular and cribriform neoplasm. Furthermore, immunohistochemistry (IHC) staining analysis was negative for Napsin A, mammaglobin, estrogen receptor (ER), and CD117. The lining of the glandular and cribriform structures also intermingled spindle cells and mostly positive $\mathrm{P} 40$, and the diagnosis of thymoma type $A B$ was made. The abdominal CT scan was normal without any metastasis. Due to consultation with a thoracic surgeon, surgery for removal of the mass was ordered after stabilizing the patient. That night she was intubated due to a decrease in $\mathrm{O}_{2}$ saturation to $70 \%$ and severe dyspnea. Unfortunately, she became asystole and died after an unsuccessful cardiopulmonary resuscitation.

\section{Discussion}

This patient had occasional dyspnea during pregnancy but she did not see a cardiologist due to fear of COVID-19 pandemic. Therefore, the latency in diagnosis and further management led to adverse outcomes.

Thymoma is a generally slow-growing and rare epithelial tumor arising from the thymus gland with no sex predilection. The etiology in most cases is unknown. Although there are rare case reports of thymoma diagnosed during pregnancy, a specific link has not been identified between them (7).

Thymoma has organotypic histology with dimorphic lymphocytes and epithelial cells (3). Type AB overexpresses p53, galectin-3, and Ki67 that might coordinately manipulate the process of development, progression, and malignant transformation (9). The tumor is mostly negative for estrogen and progesterone receptor immunoreactivity, and IHC for both ER and progesterone receptor were negative (7). In our case, Napsin A, mammaglobin, ER, and CD117 were negative and most cells were positive for $\mathrm{P} 40$.

Thymoma can be diagnosed with widely different presentations. Tuan et al. described the symptoms as myasthenia gravis, including generalized muscle weakness, ptosis, dysphagia, and dyspnea (10), or venous intraluminal extension (11). Also, the dominant chief complaint of our case was respiratory symptoms. Thymoma occurs more commonly in association with autoimmune conditions, such as myasthenia gravis, aplastic anemia, systemic lupus erythematous, and multiple endocrine neoplasia syndrome, as well as hypogammaglobulinemia (12). However, in our case, no previous medical disease was reported.

One differential diagnosis of postpartum dyspnea is pulmonary thromboembolism that was ruled out in our study with normal CT angiography, Doppler ultrasound, and a negative D-dimer. Preeclampsia is the other differential diagnosis due to pulmonary edema (8). In our patient, despite high liver enzymes, no metastasis was observed in the abdominal CT scan, and she had normal BP during pregnancy and after delivery. 


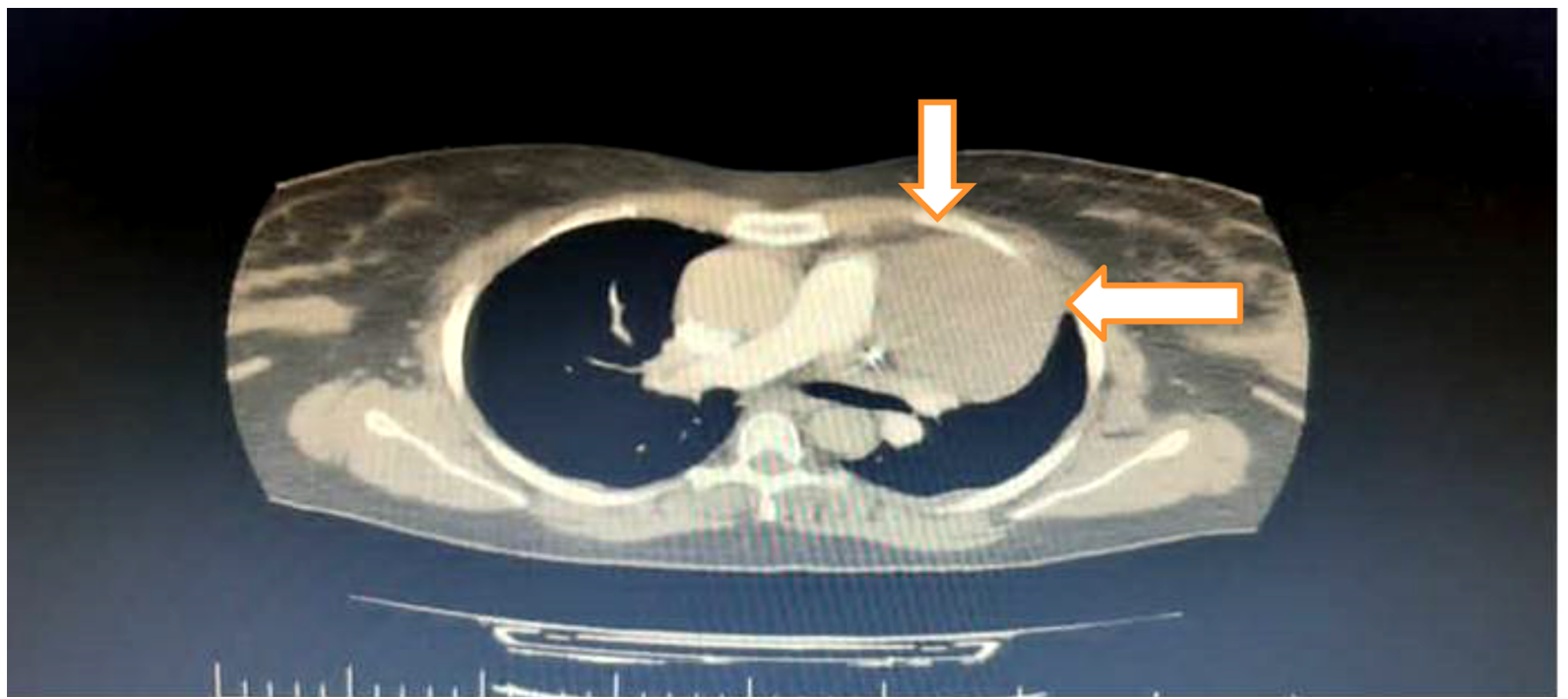

Figure 1. Computed tomographic scan shows anterior mediastinal mass; measures $64 \times 84 \mathrm{~cm}$ (white arrows).

This neoplasm has almost poor prognosis, and most reported cases are due to metastasis and recurrent disease $(13,14)$. In one case, the death was due to post cesarean section cardiac arrest (15), which was similar to our study. This is caused by rapid growth and even metastasis during pregnancy. It may be due to nonspecific suppression of the immune system that makes the tumor a tolerated entity (16).

\subsection{Conclusions}

Diagnosis and management of thymoma in pregnancy remain challenging and may deteriorate with pregnancy termination. Prenatal care during pregnancy should not be abandoned due to COVID-19 pandemic, and any symptoms similar to the physiologic changes in pregnancy should be evaluated for optimal clinical management.

\section{Footnotes}

Authors' Contribution: M.F.: Project development; S.S.: Manuscript writing; M.M.: Data collection; N.H.: Data collection; M.R.: Data collection; M.G.: Manuscript writing and final edition; All authors approved the submitted version and any substantially modified version that involves the author's contribution to the study.

Conflict of Interests: The authors have no conflict of interest.

Ethical Approval: This manuscript was performed in accordance with the Helsinki declaration. The patient's data were kept confidential.

Funding/Support: No.
Informed Consent: Written informed consent was taken from the patient in admission and from her husband after her death to publish the data.

\section{References}

1. Czeisler MÉ, Marynak K, Clarke KE, Salah Z, Shakya I, Thierry JM, et al. Delay or Avoidance of Medical Care Because of COVID-19-Related Concerns - United States, June 2020. Morb Mortal Wkly Rep. 2020;69(35). doi: 10.15585/mmwr.mm6935e3.

2. Marom EM. Imaging thymoma. J Thorac Oncol. 2010;5(10 Suppl 4):S296-303. doi: 10.1097/JTO.0b013e3181f209ca. [PubMed: 20859123].

3. Kornstein MJ. Pathology of the thymus and mediastinum. Philadelphia (PA): WB Saunders; 1995.

4. Liang X, Lovell MA, Capocelli KE, Albano EA, Birch S, Keating AK, et al. Thymoma in children: report of 2 cases and review of the literature. Pediatr Dev Pathol.2010;13(3):202-8. doi:10.2350/09-07-0672-OA.1. [PubMed: 20055684].

5. Peleg D, Zabari A, Shalev E. Relapsing thymic carcinoma during pregnancy. Acta Obstet Gynecol Scand. 1992;71(5):398-400. doi: 10.3109/00016349209021082. [PubMed: 1326220].

6. Huang CC, Lee CC. Thymoma and myasthenia gravis in pregnancy: report of a case.JFormos Med Assoc.1991;90(2):206-8. [PubMed:1678418].

7. Hechtman JF, Chepovetsky JA, Strauchen JA, Burstein DE, Beasley MB. Thymomas diagnosed during pregnancy: two cases in young women without paraneoplastic or autoimmune disease. Ann Diagn Pathol. 2012;16(5):392-6. doi: 10.1016/j.anndiagpath.2011.03.003. [PubMed: 21652248].

8. Prueksaritanond S, Ali AM, Aronu GN, Hussain N, Ganjoo A, Mirrakhimov $\mathrm{AE}$, et al. An uncommon cause of shortness of breath in a young puerpera. Case Rep Obstet Gynecol. 2013;2013:710620. doi: 10.1155/2013/710620. [PubMed: 23585977]. [PubMed Central: PMC3622346].

9. Wang Z, Li H, Cao H, Zheng J. Clinicopathological features of type AB thymoma with liver metastases. Int JClin Exp Pathol. 2014;7(12):8700-5. [PubMed: 25674235]. [PubMed Central: PMC4314039]. 
10. Tuan PA, Minh Duc N. Ectopic thymoma in the middle mediastinum: A case report and literature review. Clin Ter. 2021;172(2):94-8. doi: 10.7417/CT.2021.2291. [PubMed: 33763676].

11. Tsutsui S, Ashizawa K, Tagawa T, Nagayasu T, Hayashi T, Uetani M. Invasive thymoma with venous intraluminal extension: CT and MRI findings. Clin Imaging. 2012;36(6):854-7. doi: 10.1016/j.clinimag.2012.01.033. [PubMed: 23154023].

12. Travis WD, Brambilla E, Muller-Hermelink HK, Harris CC. World Health Organization classification of tumours. Pathology and genetics of tumours of the lung, pleura, thymus and heart. Lyon, France: IARC press; 2004.

13. Jodczyk J, Popow J. [Thymoma granulomatosum in pregnancv]. Patol Pol.1961;12:467-72. Polish. [PubMed: 14451906].

14. Massart JJ, Bishop WA. Thymoma occurring during pregnancy: a case report. Obstet Gynecol.1968;32(4):490-3.

15. Goldman KP. Malignant thymoma in pregnancy. $\mathrm{Br} J$ Dis Chest 1974;68:279-83. doi:10.1016/0007-0971(74)90052-7.

16. Ray PK, Saha S. Tumor growth versus fetal development-similarities and confusions. Adv Immun Cancer Ther. 1986;2:155-87. [PubMed: 3321946]. 